Nouvelles perspectives en sciences sociales

\title{
Éléments de critique des théories de l'action
}

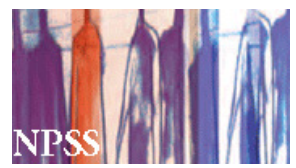

\section{Mélanie Girard}

Volume 3, numéro 1, septembre 2007

URI : https://id.erudit.org/iderudit/602465ar

DOI : https://doi.org/10.7202/602465ar

Aller au sommaire du numéro

\section{Éditeur(s)}

Prise de parole

ISSN

1712-8307 (imprimé)

1918-7475 (numérique)

Découvrir la revue

Citer cet article

Girard, M. (2007). Éléments de critique des théories de l'action. Nouvelles perspectives en sciences sociales, 3(1), 47-60. https://doi.org/10.7202/602465ar

\section{Résumé de l'article}

Malgré de nombreux développements en sciences sociales, les théories de l'action continuent de faire usage d'un appareillage conceptuel dont les concepts apparaissent comme nécessaires les uns aux autres et liés entre eux de façon égalitaire. Ces concepts, en outre, sont compris comme étant à la fois la cause et l'expression de la liberté de l'acteur. A partir de cette structure atomique, dans laquelle aucun concept n'a d'ascendant logique sur les autres, est construite et interprétée l'action humaine. Or, une réflexion approfondie nous révèle que ces concepts - conscience, rationalité, stratégie, intérêt, intention - sont de niveaux sémantiques différents, certains représentant des catégories principielles, d'autres, des catégories analytiques. Elle nous révèle aussi que les liens entre les concepts ne sont pas nécessaires. L'exercice que voici consiste à mettre en question la valeur heuristique de l'outillage conceptuel des théories de l'action; il donne cours, pour une première fois, à la création d'indicateurs pour l'analyse. 


\title{
Éléments de critique des théories de l'action
}

\author{
MÉLANIE GIRARD \\ Université Laurentienne
}

Après le fonctionnalisme de Durkheim, après le structorofonctionnalisme de Parsons, qui, tous deux, montraient comment les actions individuelles sont déterminées par les structures sociales, bon nombre de sociologies ont affirmé avec virulence la liberté de l'acteur. À titre illustratif, on peut évoquer l'interactionnisme de Goffman où l'on voit comment un individu peut « acter » de telle manière qu'il parvient à présenter de lui-même une image souhaitée et à agir sur les autres afin qu'ils le perçoivent tel qu'il entend l'être ${ }^{l}$; on peut aussi signaler l'ethnométhodologisme de Garfinkel, qui ne cesse d'affirmer que l'acteur réalise (accomplish), c'est-à-dire qu'il n'est pas déterminé par les structures sociales et qu'il peut s'autodéterminer ${ }^{2}$; on peut encore signaler l'individualisme méthodologique de Boudon qui, au nom de la liberté de l'acteur, s'oppose à tout holisme ${ }^{3}$. Ces sociologies, qui

Erving Goffman, The presentation of self in everyday life, Woodstock, New York, Overlook Press, 1973.

2 Harold Garfinkel, Studies in etbnomethodolog\%, Englewood Cliffs (NJ), Prentice-Hall, 1967.

3 Raymond Boudon, La place du désordre. Critique des théories du cbangement, Paris, PUF, [1984] 1991 ; Raymond Boudon, L'idéologie, ou l'origine des idées rectues, Paris, Fayard, 1986. 
comptent plusieurs protagonistes ${ }^{4}$, dont le pendant apparait dans d'autres disciplines ${ }^{5}$, dont les audiences sont immenses, dont l'essentiel a des échos dans la plupart des courants sociologiques ${ }^{6}$, ont rappelé avec force que l'individu, en tant que sujet, était capable d'autonomie ${ }^{7}$. Il faut dire que la position avait bien été aménagée notamment par tout le courant phénoménologique, par la sociologie wébérienne et par la science économique, qui s'est édifiée en partie sur un bomo æconomicus.

La thèse repose sur un raisonnement plutôt simple : l'acteur individuel est libre parce qu'il est rationnel; la raison lui permet d'agir en fonction de son intérêt ; cette action intéressée est possible parce qu'elle est consciente ; la conscience rend elle-même possible l'intention ; l'action menée en fonction de l'intérêt particulier est ainsi stratégie. Les concepts s'interpellent les uns les autres et il n'y a pas vraiment de point de départ dans le raisonnement. Il y a raison parce qu'il y a conscience, intention, stratégie, intérêt ; il y a conscience parce qu'il y a raison, intention, stratégie, intérêt ; il y a intention parce qu'il y a raison, conscience, stratégie, intérêt... L'appareillage conceptuel semble fait d'éléments nécessaires les uns aux autres, liés entre eux de façon

4 Aux États-Unis : Emmanuel Schlegoff, Harvey Sacks, Gail Jefferson, Don Zimmerman, Charles Goodwin... En France : Alban Bouvier, François Bourricaud, François Chazel...

Que l'on pense à la plupart des manifestations de l'approche cognitive en psychologie, que l'on pense à la théoric des jeux en économic, à la théorie des actes du langage...

"À titre d'exemples, retenons l'actionnalisme d'Alain Touraine (Critique de la modernité, Paris, Fayard, 1992 ; Le retour de l'acteur : essai de sociologie, Paris, Fayard, 1984) ; le structuralisme génétique de Pierre Bourdieu (Choses dites, Paris, Minuit, 1987; La distinction : critique sociale du jugement, Pa ris, Minuit, 1979); le fonctionnalisme stratégique de Michel Crozier et Erhard Friedberg (L'acteur et le système. Les contraintes de l'action collective, Paris, Seuil, 1977) ; le néo-fonctionnalisme de Jeffrey Alexander (Theoretical logic in sociology, vol. 1-4, Berkeley, University of California Press, 1982-1983) ; l'ethnographie institutionnelle de Dorothy Smith (The conceptual practices of power: $A$ feminist sociology of knowledge, Toronto, University of Toronto Press, 1990 ; Institutional ethnography: A sociology for people, Oxford, AltaMira Press, 2005).

Il est très facile de démontrer que Boudon n'est pas Garfinkel, que, lui-même n'est pas Goffman... Il est cependant possible de constater que ces théories de l'action se développent sur des préoccupations semblables et sur des appareillages conceptuels identiques. 
égalitaire. Et eux tous sont la cause et l'expression de la liberté de l'acteur. C'est ainsi que les théories de l'action voient les choses indépendamment de nombreux développements qui ont eu cours en philosophie analytique, en psychologie, en sciences cognitives, en analyse relationnelle, en théorie de l'intelligence artificielle et qui devraient obliger à faire plusieurs nuances dans l'usage notamment des concepts de conscience, d'intention, de raison.

On peut ainsi dessiner la modélisation des théories de l'action :

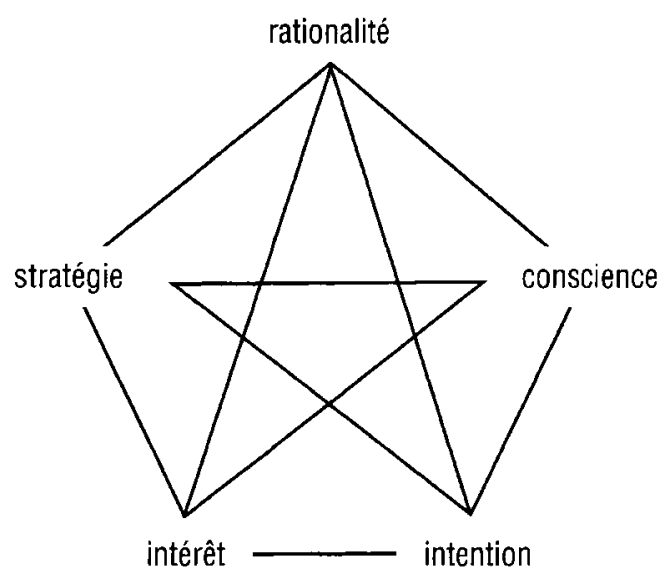

Chaque concept est attaché à tous les autres. Aucun d'entre eux n'a d'ascendant logique sur les autres. Le tout constitue une structure atomique à partir de laquelle est construite et interprétée l'action humaine.

Mais on peut s'interroger sur la justesse de cette modélisation sur laquelle reposent les théories de l'action.

Certes, il serait possible de définir de plusieurs façons chacun des concepts qui sont employés dans cet assemblage pour prendre en considération l'ensemble des désignations qu'on trouve dans les sciences humaines ; tel n'est pas l'objet de ce travail et une telle opération empêcherait de saisir la théorisation telle qu'elle est produite dans les théories de l'action. Notre travail, en effet, consiste à rappeler que les théories de l'action ont généré un ensemble de concepts. interdépendants dont les connotations se révèlent par-dessus tout dans leur interconnexion et dans les usages qui en sont faits. Il n'y a pas de 
théoricien de l'action qui mette en doute qu'un acteur social agisse en fonction de son intérêt et qui n'associe pas cette action à la rationalité. Cela ne signifie pas que nous affirmons que tout acteur agit toujours en fonction de son intérêt ${ }^{8}$.

\section{Critique de la modélisation des théories de l'action}

\subsection{Niveaux sémantiques}

On peut se demander si les concepts sont réellement égaux entre eux. Si l'on y regarde de près, on remarque que, logiquement, s'il y a intérêt, c'est qu'il y a raison, celle-ci étant la condition de possibilité de celui-là et non l'inverse ; pour percevoir un intérêt, il faut être en mesure d'établir un raisonnement par lequel se découvre un lien entre soi et le monde. Dans le même esprit, pour qu'il y ait intérêt, il faut qu'il y ait conscience car on ne peut percevoir ce qui est bien ou bon pour soi que dans la mesure où le constat est possible. Ainsi, par rapport à l'intérêt, raison et conscience sont logiquement antérieures. En même temps, il appert que, pour qu'il y ait intérêt, il faut qu'il y ait simultanément conscience et raison. Si l'une et l'autre s'appellent mutuellement pour rendre possible l'intérêt, l'intérêt, lui, n'est pas nécessaire à la raison ou à la conscience puisqu'il peut y avoir des raisonnements qui portent sur bien d'autres choses que l'intérêt et que l'on peut avoir conscience de bien d'autres choses que l'intérêt. Les concepts de conscience et de rationalité font, en réalité, figure de principes. L'intérêt en est forcément un dérivé. Dans la mesure où la stratégie correspond nécessairement à une action intentionnelle - puisqu'il ne peut y avoir de stratégie sans qu'il y ait intention - et où cette stratégie suppose l'intérêt, c'est-à-dire

* Nous nous associons davantage à un courant qui, précisément, dénonce les principes de ces logiques rationalisantes. Voir, par exemple, Simon Laflamme, Communication et émotion. Essai de microsociologie relationnelle, Paris, L'Harmattan, coll. "Logiques sociales ", 1995 ; Louis Quéré, "Iangage de l'action et questionnement sociologique ", dans Paul Ladrière, Patrick Pharo et Louis Quéré (dir.), La théorie de l'action. Le sujet pratique en débat, Paris, CNRS, 1993, p. $53-83$; Pierre Bouchard, "Théories de l'action et parcours de vie », Nowvelles perspectives en sciences saciales, vol. 1, n" 2, 2006, p. 67-115; Paul Jalbert, "Analyse du rôle de l'intention dans les échanges dyadiques", Nonvelles perspectives en sciences sociales, vol. 2, n" 1, 2006, p. 101-141. 
la capacité d'agir dans le but de satisfaire le soi, l'intention et la stratégie découlent, elles aussi, de la rationalité et de la conscience. S'il est vrai qu'il ne peut y avoir de stratégie sans conscience, il peut y avoir conscience sans qu'il y ait stratégie. Par rapport à la raison et à la conscience, stratégie, intérêt et intention constituent des dérivés potentiels. Et tout comme le lien entre conscience et rationalité en est un de possibilité, et non de nécessité, les combinaisons possibles entre stratégie, intention et intérêt sont, elles aussi, possibles, et non nécessaires ; comme le sont, d'ailleurs, les liens entre conscience ou rationalité, d'une part, et stratégie, intérêt ou intention, de l'autre.

\subsection{Toute action n'est pas consciente}

On peut aussi se demander si l'appareillage classique modélise adéquatement son objet. La conscience peut réfléchir un geste. Un geste intentionnel. Mais la plupart des gestes que pose un acteur dans une journée ne sont pas conscients; ils sont de l'ordre de l'habitude, de la routine, de l'inconscient - ce qui ne fait pas de lui pour autant un être mécanique, programmé. On exagère beaucoup quand on affirme que l'humanité n'est libre que parce qu'elle est rationnelle et consciente". L'acteur ne peut pas être toujours conscient de ce qu'il fait. L'acteur peut, par sa conscience, percevoir un intérêt, développer une intention, mettre en œuvre une stratégie. La conscience est alors à l'origine de ce qui est fait ou de ce qui est dit. Mais le rapport que l'on pourra établir entre la conscience et l'un ou l'autre de ses effets ne pourra être que probable en ce que tout ce dont on a conscience n'est pas action et tout ce qui est action n'est pas conscience.

En nous limitant à la conscience, examinons en quoi les liens que l'on peut établir entre elle et ses dérivés potentiels, qui caractérisent l'action, permettent de modéliser adéquatement l'action. L'intérêt relève de la conscience; on ne peut être intéressé que dans la mesure où l'on est conscient de ses intérêts; mais, inversement, on peut être conscient sans être intéressé. L'intention est, elle aussi, corollaire de la conscience ; mais, ici encore, l'inverse n'est pas vrai. Un acteur peut avoir conscience de quelque chose sans pour autant avoir des intentions précises. Aussi, 
l'intention peut exister sans donner lieu à l'élaboration d'une stratégie, mais l'inverse est faux : une stratégie trouve nécessairement, à sa source, une intention. On notera également qu'il faut, certes, qu'il y ait conscience pour qu'il y ait stratégie ; mais ce n'est pas parce qu'il n'y a pas stratégie qu'il n'y a pas conscience. On peut être conscient sans que cette conscience donne lieu à l'élaboration d'une stratégie. Les conséquences d'une telle information sont imposantes car, à l'inverse, on entrevoit facilement que ce n'est pas parce qu'il y a information, geste ou action qu'il y a forcément conscience. En fait, la preuve du lien entre l'information, le geste et l'action, d'une part, et la conscience, d'autre part, est autant à faire que celle de leur dissociation.

\subsection{Toute action n'est pas rationnelle}

La plupart des gestes que pose un acteur dans une journée ne sont pas conscients; ils ne sont pas non plus rationnels - ce qui ne signifie pas qu'ils ne puissent être rationnalisés par une théorie. Et, de la même façon que les liens qui existent entre la conscience et l'intérêt, l'intention ou la stratégie ne sont pas automatiques, ceux qui unissent la rationalité et l'intérêt, l'intention ou la stratégie se présentent beaucoup plus en termes de potentialité que de nécessité. L'intérêt ne donne pas obligatoirement lieu à une stratégie; on peut être intéressé sans pour autant élaborer une stratégie visant à combler cet intérêt ; pour une foule de raisons, on peut demeurer inactif devant son intérêt sans que l'inaction soit stratégie. Étre rationnel, ce n'est pas forcément être intéressé. Le fait de reconstruire a posteriori l'intention en lui attribuant des raisons logiques ne fait pas pour autant que l'intention, telle qu'elle s'est manifestée in situ, ait été rationnelle. Être rationnel, ce n'est pas nécessairement être intentionné. La stratégie appelle la raison dans la mesure où elle exige une réflexion, une élaboration en séquences; elle est un événement pluri-temporel, contrairement à l'intention qui, elle, représente davantage un mouvement psychique. Mais la raison, à son tour, n'appelle pas nécessairement la stratégie. Être rationnel, ce n'est pas obligatoirement être stratégique. 


\subsection{Conscience et rationalité ne sont pas forcément liées}

En plus de ne pas entretenir des liens exclusifs avec l'intérêt, l'intention ou la stratégie, la conscience et la rationalité, elles-mêmes, ne vont pas forcément de pair ; la conscience raisonne, mais elle n'est pas que réflexion. Le fait d'avoir conscience de quelque chose ne suppose pas pour autant qu'il soit là question de rationalité. Inversement, le fait de poser un geste ou une action à caractère rationnel n'implique pas que l'on en soit conscient : on peut, par exemple, effectuer une opération mathématique sans avoir conscience de le faire. Un acteur peut agir de façon rationnelle sans en avoir conscience ou être conscient d'une action qu'il pose qui n'est pas, en soi, rationnelle; le lien entre rationalité et conscience en est un de probabilité, et non de nécessité. Dans un même ordre d'idées, les liens qui unissent les notions de stratégie, d'intention et d'intérêt sont, eux aussi, probables, et non nécessaires : la stratégie relève de l'intention, mais, à l'inverse, l'intention ne débouche pas forcément sur la stratégie ; la stratégie peut relever d'un intérêt, comme il peut en être autrement ; l'intention peut, de la même façon, être intéressée, comme elle peut ne pas l'être.

À la lumière de ces considérations, il appert que la modélisation se résume plutôt de la façon suivante. Ainsi, elle rend compte de la nature réelle des liens entre les concepts ; elle distingue entre nécessité théorique, possibilité théorique et possibilité empirique. L'on y voit, par exemple, que la stratégie appelle nécessairement, théoriquement, la rationalité, la conscience et l'intention; que rationalité et conscience, de même que rationalité et intention sont possiblement liées au plan théorique, mais que rationalité et intérêt, comme rationalité et stratégie sont non pas possiblement, mais bien nécessairement liés au plan théorique en ce que l'intérêt et la stratégie supposent tous deux la rationalité ; qu'intention et intérêt sont possiblement liés au plan empirique en ce qu'il est possible que l'on observe qu'une intention est liée à un intérêt, mais que ce lien n'est pas automatique ; que si l'on a une intention, c'est forcément que l'on est rationnel et conscient, mais non nécessairement stratégique ou intéressé... et ainsi de suite. 


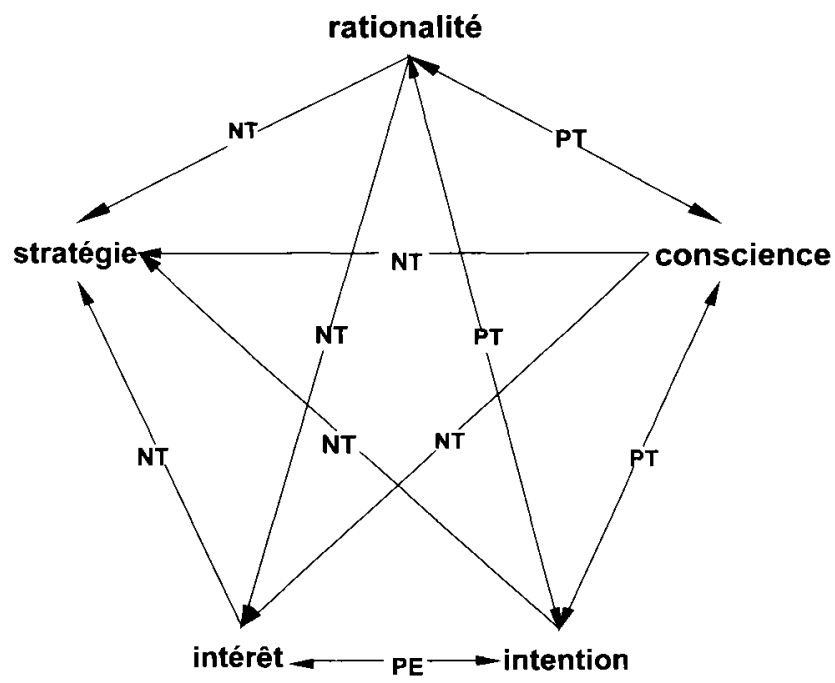

\section{Légende \\ NT = nécessité théorique \\ $\mathrm{PE}=$ possibilité empirique \\ PT = possibilité théorique}

\section{Autour de la confusion}

Cette confusion de niveaux et de relations entre les concepts donne lieu à ce qui devient, donc, une modélisation qui appelle une probabilité d'action plutôt qu'une nécessité, plutôt qu'une exhaustivité de l'action. Nous reprendrons, pour le vérifier, chacun des concepts en vérifiant son rapport à l'action. 1) L'action peut-elle être rationnelle ? La réponse à cela est oui. 2) L'action peut-elle être consciente ? La réponse à cela est encore oui. 3) L'action peut-elle être stratégique ? La réponse, ici aussi, est oui. 4) L'action peut-elle être intentionnelle? Bien entendu que si. 5) L'action peut-elle être intéressée ? Il semble tout aussi évident que l'action puisse être intéressée. Mais, à l'inverse, on peut se poser la question de savoir si l'action peut aussi ne pas être 1) rationnelle 2) consciente 3) stratégique 4) intentionnelle 5) intéressée. Et il est clair que l'action n'est pas toujours rationnelle, ou consciente, ou stratégique, ou intentionnelle, ou intéressée. Le modèle permet donc de rendre compte de probabilités d'action et non de l'ensemble des comportements, des possibilités d'action ; en cela, il se fait partiel et, du même coup, il 
surmodélise l'action humaine en cela qu'il se rend aveugle à une infinité de comportements humains.

\section{De la confusion au sein même de concepts}

Nous avons vu que les concepts ne sont pas de même niveau théorique, qu'il y a là confusion. Mais outre cette confusion de niveaux entre les concepts eux-mêmes, on trouve une confusion plus fondamentale : celle qui relève du glissement entre les deux usages qui sont faits du concept de rationalité. Il s'agit de la rationalité telle que le théoricien l'applique à l'action et de la rationalité de l'acteur lui-même. Dans cette confusion, on oublie que modéliser l'action comme rationnelle (comme le fait Weber, par exemple), ce n'est pas observer l'action comme rationnelle, ce qui soulève deux problèmes importants. Le premier problème se pose comme suit : modéliser l'action comme rationnelle, c'est définir, a priori ou a posteriori, l'action, c'est enfermer l'action dans un mode $a$ priori - ou a posteriori - plutôt que la découvrir se déployant dans ce qu'elle a de rationnel et de non rationnel. Le second consiste à prêter à l'acteur une qualité a priori. Dans ce cas, on se heurte à l'idée selon laquelle si l'action était rationnelle, c'est qu'elle serait réductible à un raisonnement purement logique, aux informations intégrées, non pas en tant que le chercheur pourrait la déclarer telle apriori ou a posteriori, mais bien en tant que l'acteur lui-même, dans sa subjectivité, ferait découler son action a posteriori de son raisonnement; ce qui pose, il va sans dire, le problème de la simultanéité de l'action et de la psyché. Si l'action découle toujours et strictement du raisonnement, alors l'acteur serait toujours conscient, réfléchi, abouti avant même de ne pouvoir se manifester, se faire être dans et par l'action. Dans un cas comme dans l'autre, les indicateurs de la rationalité seront les mêmes ; il n'importe donc pas, ici, de chercher à savoir dans quelle optique la modélisation entend rendre compte de la rationalité. De toute façon, dans la mesure où elle confond les deux, elle ne distingue pas, elle-même, clairement, l'une de l'autre.

\section{Interprétation}

Pour vérifier dans quelle mesure un modèle est valable, il importe d'en découvrir les qualités heuristiques et, donc, de le mettre en relation avec 
l'objet dont il est censé rendre compte. Les concepts dont dispose un modèle ne donnent que rarement un accès immédiat à l'empirie ; la plupart du temps, c'est en créant des indicateurs que l'on parvient à déterminer dans quelle mesure il y a adéquation entre le modèle et les données.

Pour la conscience et la rationalité, le problème suivant se pose : comme le soulignent Mead et Bateson ${ }^{10}$, on ne peut les observer que par dérivation puisque leur degré d'abstraction est très élevé - ce qui nous renvoie au problème de la hiérarchie des niveaux sémantiques. Cela, toutefois, n'interdit pas l'opération.

Si l'on effectue cette opération en fonction de la raison, il nous semble que l'on devrait repérer la rationalité si l'on trouve la trace 1) d'une organisation logique d'informations, 2) d'un enchaînement logique de gestes ou d'actions, 3) d'une concordance entre des gestes ou des actions, d'une part, et des informations, d'autre part - une discordance pourrait témoigner d'une mauvaise foi ou d'une stratégie et, donc, renvoyer elle aussi à la rationalité. Cette logique qui doit être observée n'est pas obligatoirement objective en ce sens qu'elle peut ne pas apparaitre immédiatement à l'observateur muni de ses instruments analytiques; elle est nécessairement subjective puisqu'elle se manifeste selon un schéma congruent chez un acteur particulier. Ces traces sont donc le signe de l'existence de la raison. Dans cet esprit, par conséquent, tout ce qui ne donne pas à apercevoir cette trace, de même que tout ce qui indique qu'elle n'existe pas, doit inviter à mettre en doute le caractère rationnel des informations, des gestes ou des actions qui se révèlent à l'observateur. Ainsi, tous les signes d'une non-organisation logique, d'un non-enchaînement logique, d'une non-concordance entre l'information et les actions ou les gestes - sauf découverte, à un second degré, de mauvaise foi ou de stratégie - représentent des éléments de contestation de la thèse de la rationalité de l'action humaine, du moins, dans son caractère universel ${ }^{11}$.

I" George Herbert Mead, L'esprit, le soi et la société, Paris, PUF, 1963 ; Gregory Bateson, Steps to an ecology of the mind, Boulder, Paladin Books, 1973.

Voir Mélanie Girard, Simon Laflamme et Pascal Roggero, «L'intention est-elle si universelle que ne le prétendent les théories de l'action? ", Nouvelles perspectives en sciences sociales, vol. $1, \mathrm{n}^{\prime \prime} 2,2006$, p. 115-148. 
Si l'on effectue l'opération de rapprochement entre concept et indicateurs en fonction de la conscience, on peut évoquer l'un des principes que la phénoménologie a bien établis de Husserl à Sartre et Merleau-Ponty. Ce principe veut, en effet, que la conscience n'existe pas en soi, qu'elle ne constitue pas un récipient que l'on remplit au cours de la vie; il veut, bien au contraire, que la conscience soit toujours conscience de quelque chose. S'il en est ainsi, l'analyse devrait consister à repérer des informations dont l'acteur a conscience, donc à établir que l'acteur a conscience de l'information qui a été relevée; dans le même esprit, elle pourrait avoir pour fin de détecter les actions ou les gestes qui sont posés dont on peut affirmer que l'acteur en a conscience.

Pour établir ce lien entre l'information, le geste ou l'action, d'une part, et la conscience, d'autre part, on peut se référer à des positions de principe et reconnaitre, par exemple, qu'une stratégie est forcément consciente, sinon elle n'est pas stratégie : comment, en effet, un acteur peut-il développer une stratégie dont il n'aurait pas conscience ! - la notion de stratégie inconsciente est l'un de ces avatars des théories de l'action qui témoignent de leur incapacité à rendre compte de phénomènes beaucoup plus complexes que ceux que peut observer leur modèle ${ }^{12}$. On pourra encore souligner que l'intention, en tant qu'inscrite dans une démarche consciente, est tributaire d'un procédé correspondant à l'affirmation d'un projet, minimalement; le discours de l'acteur pourra également comporter la répétition, le retour-sporadique ou régulier - sur l'affirmation présentée ; il pourra même donner cours à une démarche qui effectue cette répétition même si des informations contraires sont émises. (Nous ne tenons pas compte, ici, des notions d'intention inconsciente ou d'intention voilée, pour la simple raison que la première témoigne de la même incapacité que celle qui a été évoquée plus haut, soit celle de la théorie de l'action à rendre compte de phénomènes qui sont plus complexes que ce que son appareillage permet d'expliquer, et que la seconde se rapporte plutôt à la stratégie et ne relève donc plus, dès lors, de la stricte intention.) Quelles que soient les positions de principes qui seront isolées, il n'en reste pas moins que la difficulté à faire la démonstration de la conscience d'un objet observé - information, geste ou action - constitue en soi une invitation 
à la suspicion à l'égard des théories de l'action, et surtout à l'endroit de leur prétention à l'universalité.

Les concepts de stratégie, d'intention et d'intérêt sont, eux aussi, abstraits ; mais moins que ne le sont les deux précédents. Ces trois concepts peuvent donner accès et à la conscience et à la rationalité. Ils donnent accès à la conscience en ce sens que leur objet peut faire état d'un acte conscient, comme c'est nécessairement le cas pour la stratégie et l'intention, comme c'est aussi le cas pour l'intérêt dès lors qu'il est subjectif; l'intérêt objectif, c'est-à-dire tel que défini par une théorie ou une idéologie, peut correspondre à l'intérêt subjectif, mais cela n'est aucunement nécessaire, si l'intérêt n'est pas transparent à l'acteur, s'il est indéfini, c'est tout simplement que l'on ne peut pas en avoir conscience, et donc qu'il ne peut constituer un intérêt. Ils donnent accès à la rationalité, comme c'est forcément le cas pour une stratégie, comme c'est possiblement le cas pour l'intention; la perception en elle-même de l'intérêt n'est pas rationnelle, c'est l'acte qui est posé en fonction de l'intérêt qui, lui, est rationnel, et il est alors stratégie. Ces trois concepts, toutefois, désignent par eux-mêmes une réalité, et cela, de façon plus immédiate que ne le font la conscience et la rationalité.

Pour observer empiriquement l'intention, diverses voies s'offrent au chercheur. Il faut au préalable reconnaitre que l'intention est projet. C'est dans cet espace intellectuel que l'a comprise la sociologie Verstehen et c'est dans cette confusion que l'a comprise la phénoménologie existentielle. On trouve, par exemple, la trace d'une intention quand on peut montrer qu'une information émise ou qu'un geste posé est la conséquence d'un projet antérieur, que le projet précède un événement communicationnel ou qu'il surgisse en lui. On trouve la trace d'une intention quand une information émise ou un geste posé annonce un propos ou une action ultérieure. Cela dit, ce n'est pas parce qu'il y a eu intention qu'il y aura action, ou, dans le même esprit, ce n'est pas parce qu'il y a action qu'il y a eu intention. Il y a intention si l'on peut observer que, par exemple, dans une suite de tours de parole, le discours suit davantage le projet d'un énonciateur qu'il ne 
relève de la dynamique entre les interlocuteurs ${ }^{13}$. Il faut conclure à un effet d'intention si l'on peut reconstruire a posteriori un projet en examinant une trame discursive. Pour observer la stratégie, non seulement faut-il repérer les indices d'une intention, mais il faut en plus repérer un plan. Ce plan peut bien simplement correspondre à un rapport de convergence entre des moyens et des fins comme on le note dans la méthode wébérienne. Il peut aussi apparaitre dans une séquence d'actions ou de propos orientés vers une fin, mais il faudra que cette séquence d'actions ou de gestes permette de conclure à un enchaînement. La stratégie sera d'autant plus active que, lors de l'événement communicationnel, le stratège évoluera en marge de la dynamique en cours. On note la trace de l'intérêt dans le fait que l'acteur puisse évoquer un motif par lequel il entrevoit quelque avantage, que cela corresponde soit à une atténuation des coûts anticipés, soit à un bénéfice. Dans tous les cas contraires, et cela, pour ce qui a trait aussi bien à l'intention, à la stratégie qu'à l'intérêt, l'observation signale le cas d'événements qui ne correspondent pas à ceux que peut repérer la théorie de l'action.

\section{Conclusion}

Ces constats sont importants. Ils obligent à repenser l'appareillage des théories de l'action. Ils permettent, pour la première fois, de tirer les conséquences logiques de ses postulats. Ils distinguent les principes ou méta-concepts des concepts. Ils dotent d'indicateurs pour l'empirie. Ils invitent à l'opérationnalisation et, par voie de conséquence, à une manipulation subséquente du modèle, le rapport entre la théorie et l'objet de la théorie étant nécessairement récursif. Ils rappellent qu'il n'est plus possible d'appliquer aveuglément un modèle dont l'absence d'empirie a permis que soit occultée toute une dimension de l'action

La thèse de l'intention a bien été mise à l'épreuve, entre autres, par les travaux de Mélanie Girard et de Paul Jalbert : Mélanie Girard, Relations bumaines et production d'information: l'échange comme abjet d'étude d'une approche relationnelle, mémoire de maitrise, Sudbury, Université Laurentienne, 2004 ; Paul Jalbert, «Analyse du rôle de l’intention dans les échanges dyadiques ", Nowvelles perspectives en sciences sociales, vol. 2, n" 1, 2006, p. $101-141$. 
humaine qui ne s'explique pas en termes d'intention ou d'intérêt ou de stratégie ou de rationalité ou de conscience. Ils ouvrent la voie à des modélisations plus complexes, plus exhaustives, plus humaines.

\section{Bibliographie}

Alexander, Jeffrey, Theoretical logic in sociology, vol. 1-4, Berkeley, University of California Press, 1982-1983.

Bateson, Gregory, Steps to an ecology of the mind, Boulder, Paladin Books, 1973.

Bouchard, Pierre, "Théories de l'action et parcours de vie ", Nouvelles perspectives en sciences sociales, vol. 1, $\mathrm{n}^{\circ} 2,2006, \mathrm{p} .67-115$.

Boudon, Raymond, La place du désordre. Critique des théories du changement, Paris, PUF, [1984] 1991

Boudon, Raymond, L'idéologie, ou l'origine des idées recues, Paris, Fayard, 1986.

Bourdieu, Pierre, Choses dites, Paris, Minuit, 1987.

Bourdieu, Pierre, La distinction : critique sociale du jugement, Paris, Minuit, 1979.

Crozier, Michel et Erhard Friedberg, L'acteur et le système. Les contraintes de l'action collective, Paris, Seuil, 1977.

Garfinkel, Harold, Studies in etbnomethodology, Englewood Cliffs (NJ), Prentice-Hall, 1967.

Girard, Mélanie, Relations bumaines et production d'information: l'échange comme objet d'étude d'une approche relationnelle, mémoire de maitrise, Sudbury, Université Laurentienne, 2004.

Girard, Mélanie, Simon Laflamme et Pascal Roggero, "L'intention est-elle si universelle que ne le prétendent les théories de l'action?", Nouvelles perspectives en sciences sociales, vol. 1, n" 2, 2006, p. 115-148.

Goffman, Erving, The presentation of selfin everyday life, Woodstock, New York, Overlook Press, 1973.

Jalbert, Paul, "Analyse du rôle de l'intention dans les échanges dyadiques ", Nouvelles perspectives en sciences sociales, vol. 2, n" 1, 2006, p. 101-141.

Laflamme, Simon, Communication et émotion. Essai de microsociologie relationnelle, Paris, L'Harmattan, coll. « Logiques sociales »,1995.

Mead, George Herbert, L'esprit, le soi et la société, Paris, PUF, 1963.

Quéré, Iouis, "Langage de l'action et questionnement sociologique ", dans Paul Ladrière, Patrick Pharo et Louis Quéré (dir.), La théorie de l'action. Lee sujet pratique en débat, Paris, CNRS, 1993, p. 53-83.

Smith, Dorothy, Institutional ethnography: A sociology for people, Oxford, AltaMira Press, 2005.

Smith, Dorothy, The conceptual practices of power: A feminist sociology of knowledge, Toronto, University of Toronto Press, 1990.

Touraine, Alain, Critique de la modernité, Paris, Fayard, 1992.

Touraine, Alain, Le retour de l'acteur : essai de sociologie, Paris, Fayard, 1984. 